polycythaemia was due to treatment with radio-phosphorus. B. Modan and A. M. Lilienfeld ${ }^{6}$ studied the records of 1,222 patients in the U.S.A. and found that acute leukaemia occurred in $11 \%$ of patients treated with radio-phosphorus and in $8.9 \%$ of patients treated with $x$ rays. They concluded that the incidence was related to the total dose of radiation received by the patient. The only contrary finding is that of K. E. Halnan and M. H. Russell, ${ }^{7}$ who had not met a case of acute leukaemia among their 107 patients treated with phosphorus-32. The latest publication on this matter is again from Szur and Lewis, ${ }^{8}$ who have recorded the haematological complications in a group of 169 patients treated with radio-phosphorus. Complications appeared in 18 patients $(11 \%)$; four had acute leukaemia, 12 had myelesclerosis, and two developed depression of bone-marrow. It is interesting that Perkins and his colleagues ${ }^{5}$ also found myelosclerosis in $4 \%$ of their patients ; it may be a complication that is not dependent on treatment.

The practical effect of all this information on the management of polycythaemia has been to show that both radiophosphorus and chemotherapy should be available for treatment. Radio-phosphorus is usually administered in a single intravenous dose, of 4 to 7 microcuries, judged on the severity of the disease and the weight of the patient. Doses are repeated when the blood estimations show it to be necessary. The present methods of treatment by chemotherapy have been described by Perkins and his colleagues, and new and better methods may soon be found. Whichever method is used initially, serious symptoms should be controlled by venesection, since both phosphorus-32 and chemotherapy take four to six weeks to be effective. A few mild cases can be controlled by venesection alone for a time. Radiophosphorus has some advantages despite the risk of leukaemia, for this risk is small and sometimes worth accepting, especially for older patients. On the other hand, the recognition of the risk should strengthen the case for the use of chemotherapy in the relatively few patients who develop the disease young, say below the age of 50 years. A few patients will not respond satisfactorily to either radio-phosphorus or chemotherapy alone, and a combination will then be needed. Certainly present therapeutic methods allow patients with polycythaemia vera to be offered a good prognosis with retention of normal activity for many years.

\section{Stones from the Kidneys}

Ureteric calculi nearly always originate in the kidneys, rarely in the ureters, and about half are subsequently passed spontaneously. Indeed, an attack of renal colic often means that this has happened, and patients may derive some comfort by being allowed to share this knowledge.

Pain caused by a calculus in the proximal part of the right ureter may resemble that of biliary colic and cholecystitis, and if the stone becomes impacted the ipsilateral kidney usually becomes tender and enlarged. Such ureteric calculi seldom pass spontaneously. The pain caused by a calculus in the distal part of a ureter may resemble the pain of appendicitis. The majority of such calculi pass spontaneously. In a series of 447 cases of ureteric colic caused by calculi $^{1}$ expectant treatment proved to be effective in $60 \%$ when the calculi had reached the middle and distal thirds of the ureter, but when the stones were in the proximal third expectant treatment was successful in only $12 \%$ of cases.

An exact diagnosis depends on adequate radiology, for blood clots and debris from renal or ureteric tumours or from necrotic renal papillae, or bleeding which complicates anticoagulant therapy or systemic bleeding states, may produce similar symptoms. Plain $x$-ray films of good quality may be expected to show calculi no greater than $2 \times 1 \mathrm{~mm}$., but may not do so if the patient is unduly obese, if the calculus is relatively non-radio-opaque, ${ }^{2}$ or if it is superimposed on an equally radio-opaque skeletal background. It is easy to overlook a small relatively non-opaque urate or uric-acid calculus in the middle part of a ureter and subject the patient with pain in the right iliac fossa and vomiting to emergency appendicectomy. ${ }^{3}$

Irvine $\mathrm{Smith}^{4}$ has recently recorded a personal assessment of the value of emergency excretion urography in patients with ureteric colic, a technique introduced 30 years ago ${ }^{5-7}$ but not extensively practised in the United Kingdom, and has pointed out that delayed excretion of the contrast medium, an enlarged nephrogram, and delayed filling (up to 24 hours or more) of a dilated collecting tract are characteristic radiographic signs. Such emergency urograms may be invaluable when the clinical diagnosis is in doubt, and moreover may provide identification of the cause and site of the obstruction in a high proportion of cases. However, it is not always easy for busy diagnostic $x$-ray departments to cope with such emergencies at short notice, and the presence of intestinal gaseous distension may impair the quality of the films. Nevertheless, there can be little doubt that the method is valuable and should be used whenever possible. Miles Fox and his colleagues ${ }^{8}$ in Leeds used it in 119 of 292 patients with ureteric calculi and agree about its value, especially when delayed films, up to 24 hours, and oblique views after evacuation of the bladder are taken. A doubtful opacity, which might otherwise be difficult to recognize as a calculus, forms the apex of a filled, obstructed ureter.

The patient suffering from the acute pain of ureteric colic usually requires pethidine or morphine and atropine by injection. In the subsequent management each case has to be considered on its own merits. For example, a patient with a calculus impacted in the ureter of a solitary kidney producing obstructive anuria may require dialysis and early operative removal of the stone, whereas a calculus which remains in a ureter for weeks, or even months, without causing persistent pain, infection, or hydronephrosis (demonstrable by repeated excretion urography at first at six-week intervals) may be left for a year or more. It is rare for a ureteric calculus with a diameter greater than $6 \mathrm{~mm}$. to pass spontaneously. Surgical intervention was undertaken by the Leeds group in $70 \%$ of their cases: transurethral instrumentation in 118, uretero-lithotomy in 109, and nephroureterectomy in 1 . However, transurethral instrumentation, consisting in the main of ureteric catheterization, is usually carried out as a diagnostic rather than a therapeutic

\footnotetext{
Guinn, G. A., and Leader, A. J., Amer. Surg., 1958, 24, 939.

Cohen, G., S. Afr. med. F., 1958, 32, 1089.

${ }^{3}$ Dourmashkin, R. L., f. Urol. (Baltimore), 1945, 54, 245

Smith, I., Brit. F. Surg., 1966, 53, 93.

5 Hellmer, H., Acta radiol. (Stockh.), 1935, 16, 51.

Wulff, H. B., ibid., 1935, 16, 77.

- ibid., 1936, Suppl. 32.

8 Fox, M., Pyrah, L. N., and Raper, F. P., Brit. f. Urol., 1965, 37, 660

9 Sandegård, E., Acta chir. scand., 1958, 116, 44.
} 
procedure. It may be tempting to conclude that subsequent passage of a calculus after ureteric catheterization is a consequence of the manœuvre, but such a conclusion is unjustified.

There may be a place for giving osmotic diuretics, such as intravenous infusions of urea. The "stone basket" for extracting the calculus has limitations: its use is restricted to calculi in the distal third of the ureter, and in the patient with impacted stone-the very case that may require the most careful and precise treatment-it is frequently impossible to get the basket beyond the stone, and injudicious manipulation may be followed by damage to the ureter and subsequent stenosis of it. Whatever method is employed for the management of ureteric calculi, patients should remain under regular surveillance, for recurrent calculi are apt to develop in about $20 \%$ of cases.

\section{Irradiation of Food}

In answer to a parliamentary question on 4 July Mr. Kenneth Robinson, Minister of Health, announced that he and other Ministers concerned had accepted the Report of the Working Party on Irradiation of Food. ${ }^{12}$ Regulations will now be introduced under the Food and Drugs Act to prohibit the irradiation of food and food products intended for human consumption, and the sale in this country of food which has been irradiated. But this legislation will in fact result in the setting up of machinery for obtaining official approval for specific processes. A scrutinizing body will be established to advise on applications for exemptions from the prohibition. The equivalent of a " permitted list" might appear, as is the case with certain classes of food additives.

Similar prohibitive legislation was made in the Federal Republic of Germany in 1959 and is under consideration in other countries. Another approach to legislation is to invoke existing regulations such as those governing food additives, with or without explicit mention of irradiated foods or definition of food irradiation as a food additive. In Canada two irradiated foods have already been approved for human consumption, potatoes and onions, and the requests for approval were dealt with under the food additive regulations. The first commercially operated food irradiation plant, situated near Montreal, began operation in 1965, and is used for the treatment of potatoes to inhibit sprouting. Food irradiation is also dealt with as a food additive in the U.S.A., where wheat, potatoes, and bacon have already been cleared by the Food and Drug Administration, although no fully commercial processes are yet operating. However, a pilot plant for the disinfestation of grain by irradiation has been completed there, and another is planned in Turkey.

International organizations are studying legislative procedures in different countries so as to give guidance on international trade in irradiated products, and a joint report on the technical basis for legislation has just been published. ${ }^{3}$ In the meantime research into various aspects of food irradiation will continue in Great Britain, ${ }^{4}$ with particular interest in processes aimed at extending the refrigeration life of meat and fish and

\footnotetext{
1 Report of the Working Party on Irradiation of Food, 1964. Ministry of Health, H.M.S.O.

2 Brit. med. F., 1964, 2, 1088.

3 The Technical Basis for Legislation on Irradiated Food. Report of joint F.A.O./I.A.E.A./W.H.O. Expert Committee, 1966. H.M.S.O.

- Brit. med. F., 1964, 1, 1330.
}

at the elimination of salmonellae from certain imported foods. ${ }^{4}$

\section{Boric Acid and Babies}

Boric (or boracic) acid and borax (sodium borate) may cause serious harm to infants, and many deaths have resulted from their use. Now a statement from the British Medical Association is published this week (p. 233) advising doctors not to prescribe them for infants.

The symptoms of intoxication are diarrhoea and vomiting, cramp, haematemesis, melaena, a red beefy rash covering the entire body, and peripheral circulatory failure. Necropsy shows cloudy swelling of the kidney, central necrosis of the liver, and haemorrhagic enteritis. ${ }^{1}$ It is easy to see how the diagnosis of gastro-enteritis could be made because of the diarrhoea and vomiting when in fact the symptoms are due to boric acid poisoning. When in doubt a simple test should give the answer. The urine is acidified by dilute hydrochloric acid, and turmeric paper is inserted ; the paper becomes red, changing to dark green on adding ammonia or dilute sodium hydroxide.

R. B. Goldbloom and A. Goldbloom ${ }^{2}$ described four cases of boracic acid poisoning and reviewed 109 others. The overall mortality was $55 \%$, but in infants under 1 year old it was $70 \%$. They emphasized that there may be considerable absorption from broken surfaces and mucous membranes. L. C. Wong and colleagues ${ }^{3}$ reported more recently on 11 infants who accidentally received boric acid in the diet. Five of them died. These authors, as others have done, question whether boric acid is of sufficient therapeutic value to justify these occasional grave mishaps. The 1966 edition of the British National Formulary includes borax glycerin, B.P.C., in the "Dental Practitioners Formulary," and boric acid ear drops, B.P.C. for " the later toilet of the ear."

Borax glycerin is a highly dangerous preparation to put in a baby's mouth because of the absorption from the mucous membrane. Mothers soak the dummy in this mixture for so-called teething, and its use for this purpose should be stopped. In any case the use of glycerin or similar material on a dummy is thoroughly undesirable for older babies, because of its disastrous effect on their teeth. There is in fact no place for any local application to palliate the troubles of teething. It seems unlikely that boric acid ear drops for the toilet of the ear will present danger, but their value is at least questionable. There is the ever-present danger that someone will attempt to treat acute otitis media with ear drops instead of giving an antibiotic, such as penicillin, by injection or by mouth.

Boric acid crystals should never be sprinkled on to the napkin for the treatment of a napkin rash because of the serious risk of absorption. Nor, for the same reason, should napkins be washed out in boric acid solution. Boric acid should not be applied in any form to a napkin rash except only as a constituent of dusting powder : $5 \%$ boric acid in talc is not absorbed through the intact skin. ${ }^{4-6}$ This substance is added to the powder to neutralize the alkalinity due to the

\footnotetext{
1 Brooke, C., and Boggs, T., Amer. F. Dis. Child., 1951, 82, 465.

Goldbloom, R. B., and Goldbloom, A., f. Pediat., 1953, 43, 631. Wong, L. C., Heimbach, M. D., Truscott, D. R., and Duncan, B. D.,
Canad. med. Ass. F., 1964, 90, 1018 .

4 Fisher, R. S., Lancet, 1954, 2, 494

5 Vignec, A. J., and Ellis, R., Amer. F. Dis. Child., 1954, 88, 72

6 Johnstone, D. E., Basila, N., and Glaser, J., F. Pediat., 1955, 46, 160

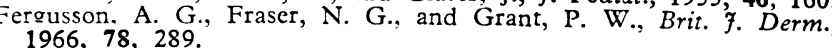

8 Warin. R. P., and Faulkner, K. E., ibid., 1961, 73, 445.
} 